\title{
A Geometric Study of the Hypergeometric Function with Imaginary Exponents
}

Takeshi Sasaki and Masaaki Yoshida

\section{CONTENTS}

\section{Introduction}

2. Local Solutions and Relations Among Them

3. Three Circles

4. Fundamental Domains

5. Numerical Experiments

6. Conclusion and Questions

Acknowledgements

References

Keywords: hypergeometric function, hypergeometric equation, Schwarzian derivative, Schwarz $s$-function, Schwarz reflection principle, Schottky group, connection matrix
The Schwarz map defined by the ratio of two solutions of the hypergeometric equation has been studied mainly when the exponents are real. In this paper, we study this map when the exponents are purely imaginary, a case that has been neglected for over a hundred years. A fundamental domain in the source plane and that in the target plane are constructed; the Schwarz map restricted on these domains is conformally isomorphic and the whole map can be recovered by this restriction through repeated use of the Schwarz reflection principle. We investigate the shape of these fundamental domains both analytically and numerically, and conclude with open questions.

\section{INTRODUCTION}

We consider the hypergeometric differential equation

$$
x(1-x) u^{\prime \prime}+(c-(a+b+1) x) u^{\prime}-a b u=0
$$

and (any) two linearly independent solutions $u_{1}$ and $u_{2}$. The (multi-valued) map

$s: \mathbb{C}-\{0,1\} \ni x \longmapsto u_{1}(x): u_{2}(x) \in \mathbb{P}^{1}:=\mathbb{C} \cup\{\infty\}$

is often called a Schwarz map (or Schwarz's $s$-map). The local behavior of this map can be determined by the local exponents

$$
\{0,1-c\}, \quad\{0, c-a-b\}, \quad\{a, b\}
$$

at $0,1, \infty$, respectively. What happens about the map $s$ when the exponents are real is well-known (for the story of Schwarz's triangles refer to [Yoshida 1997]); much less known is the case of nonreal exponents. Though F. Schilling [1894; 1895] studied the map $s$ for general exponents including purely imaginary ones, and pointed out that the map behaves much differently from the real case, no further detailed study has been made since.

In this paper, we restrict ourselves to the case of purely imaginary exponents, and find a domain $F_{x}$ 
in the $x$-plane and a domain $F_{s}$ in the $s$-plane so that the restricted map

$$
\left.s\right|_{F_{x}}: F_{x} \rightarrow F_{s}
$$

is conformally isomorphic and the whole $s$ can be recovered by $\left.s\right|_{F_{x}}$ through Schwarz's reflection along the sides of $F_{x}$. By the help of these fundamental domains, we describe the map $s$ restricted to the upper half $x$-plane. Though its image is bounded by three circles, it is not the complement of three discs in $\mathbb{P}^{1}$; it covers the whole $\mathbb{P}^{1}$ infinitely many times. On the contrary, the image of the upper half $s$-plane under the map $s^{-1}$ is the complement of three topological discs in $\mathbb{P}^{1}$.

The domain $F_{s}$ is bounded by three segments and three semicircles, whereas the domain $F_{x}$ is bounded by three segments and three curves. These curves are not parts of circles as we prove; however, as several numerical experiments shows they are very close to (parts of) circles. We are eager to know about these curves; in hope of getting a hint we numerically traced them.

\section{LOCAL SOLUTIONS AND RELATIONS AMONG THEM}

We recall some standard facts; see [Iwasaki et al. 1991, pp. 38, 39, 114], for example. For given $a, b, c$, put

$$
\lambda=1-c, \quad \mu=c-a-b, \quad \nu=a-b,
$$

and write

$$
(a, n)=a(a+1) \cdots(a+n-1),
$$

where $n$ is a positive integer. The hypergeometric series $F(a, b, c ; x)$ is

$$
F(a, b, c ; x)=\sum_{n=0}^{\infty} \frac{(a, n)(b, n)}{(c, n)(1, n)} x^{n} .
$$

Around $x=0$, local solutions of the hypergeometric equation can be given by

$$
\begin{aligned}
& f_{0}(x ; 0)=F(a, b, c ; x), \\
& f_{0}(x ; \lambda)=x^{\lambda} F(a-c+1, b-c+1,2-c ; x),
\end{aligned}
$$

and around $x=1$ by

$$
\begin{aligned}
& f_{1}(x ; 0)=F(a, b, a+b-c+1 ; 1-x), \\
& f_{1}(x ; \mu)=(1-x)^{\mu} F(c-a, c-b, c+1-a-b ; 1-x),
\end{aligned}
$$

These solutions are related by

$$
\left(f_{0}(x ; 0), f_{0}(x ; \lambda)\right)=\left(f_{1}(x ; 0), f_{1}(x ; \mu)\right) P,
$$

where

$$
P=\left(\begin{array}{ll}
D & C \\
B & A
\end{array}\right)
$$

for

$$
\begin{aligned}
& D=\frac{\Gamma(c) \Gamma(c-a-b)}{\Gamma(c-a) \Gamma(c-b)}, \quad C=\frac{\Gamma(2-c) \Gamma(c-a-b)}{\Gamma(1-a) \Gamma(1-b)}, \\
& B=\frac{\Gamma(c) \Gamma(a+b-c)}{\Gamma(a) \Gamma(b)}, \quad A=\frac{\Gamma(2-c) \Gamma(a+b-c)}{\Gamma(a-c+1) \Gamma(b-c+1)},
\end{aligned}
$$

and where $\Gamma$ denotes the Gamma function. Define two Schwarz maps

$$
s_{0}=\frac{f_{0}(x ; \lambda)}{f_{0}(x ; 0)} \quad \text { and } \quad s_{1}=\frac{f_{1}(x ; \mu)}{f_{1}(x ; 0)}
$$

which are related as

$$
s_{0}=\frac{A s_{1}+C}{B s_{1}+D} \quad \text { and } \quad s_{1}=\frac{D s_{0}-C}{-B s_{0}+A} .
$$

Note the local behavior

$$
\begin{array}{ll}
s_{0} \approx x^{\lambda} & \text { around } x=0, \\
s_{1} \approx(1-x)^{\mu} & \text { around } x=1 .
\end{array}
$$

\section{THREE CIRCLES}

From now on we assume that the exponents $\lambda, \mu$, and $\nu$ are purely imaginary (and distinct from zero, of course). Changing the unknown $u$ of the hypergeometric equation by multiplying a suitable power of $x$ and $1-x$, we get the equation

$$
u^{\prime \prime}+p(x) u=0
$$

where

$$
4 p(x)=\frac{1-\lambda^{2}}{x^{2}}+\frac{1-\mu^{2}}{(1-x)^{2}}+\frac{1+\nu^{2}-\lambda^{2}-\mu^{2}}{x(1-x)} .
$$

Note that the rational function $p(x)$ is defined over the reals. Thus for any real point $x(\neq 0,1)$, there are two linearly independent solutions of the hypergeometric equation such that their ratio takes real values along small real interval near $x$. Since a(ny) linear fractional transformation takes circles (we regard lines as circles passing through $\infty$ ) to circles, the images under a(ny) Schwarz map of the intervals

$$
(-\infty, 0), \quad(0,1), \quad(1,+\infty)
$$

are circles. 

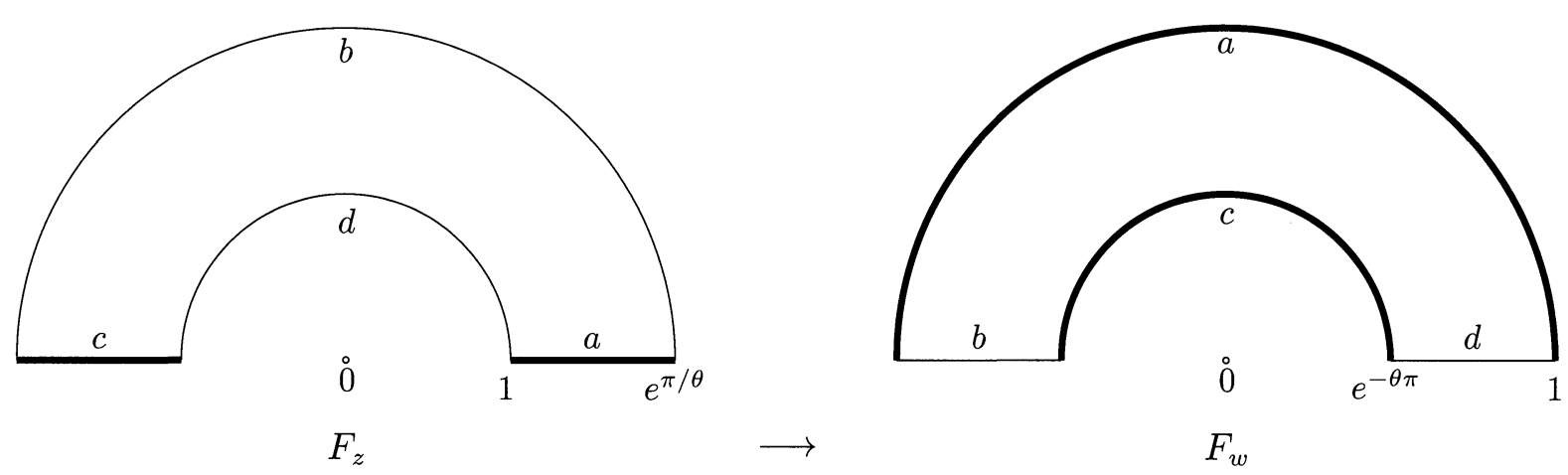

FIGURE 1. Two fans $F_{z}$ and $F_{w}$, with $\theta>0$.

We will determine these circles. Since, for example, the map $s_{0}$ is asymptotically equal to the power function $x^{\lambda}$ around the origin, we recall the behavior of power functions.

\section{Maps Defined by Power Functions}

The behavior of the power function

$$
w:=z^{\alpha}=\exp (\alpha \log z),
$$

where $\alpha=i \theta$ with $\theta \in R-\{0\}$, can be described as follows. If we take $\log 1=0$, then the real positive line is mapped to the unit circle, and the upper half plane is mapped on the ring domain bounded by the unit circle and the circle of radius $\exp (-\theta \pi)$. Cut the image ring along the real axis and we get a fan $F_{w}$. The inverse image is also a fan; call it $F_{z}$. Then the $\infty$-to- $\infty$ map $z \mapsto w$ is made (through the reflection principle) by the one-to-one map $F_{z} \rightarrow$ $F_{w}$; the line segments are mapped to circular arcs and the circular arcs to line segments (Figure 1). Any small neighborhood of $z=0$ in the upper halfplane is mapped onto the ring domain above.

\section{Arrangement of the Three Circles}

Put

$$
\lambda=i \theta_{0}, \quad \mu=i \theta_{1}, \quad \nu=i \theta_{2},
$$

so that

$$
\begin{aligned}
& a=\frac{1}{2}-\frac{1}{2} i\left(\theta_{0}+\theta_{1}+\theta_{2}\right), \\
& b=\frac{1}{2}-\frac{1}{2} i\left(\theta_{0}+\theta_{1}-\theta_{2}\right), \\
& c=1-i \theta_{0} .
\end{aligned}
$$

Convention. Unless otherwise stated we always take a branch of the logarithm function such that the log of a positive real number is real.
We will study the behavior of the Schwarz map on the upper half $x$-plane. Since this map takes the three real intervals to three circles, it is natural to ask the arrangement of the three circles. They would not intersect. Then note that there are two ways topologically to put three disjoint circles on the sphere: like a dartboard and like a pig nose. See Figure 2.
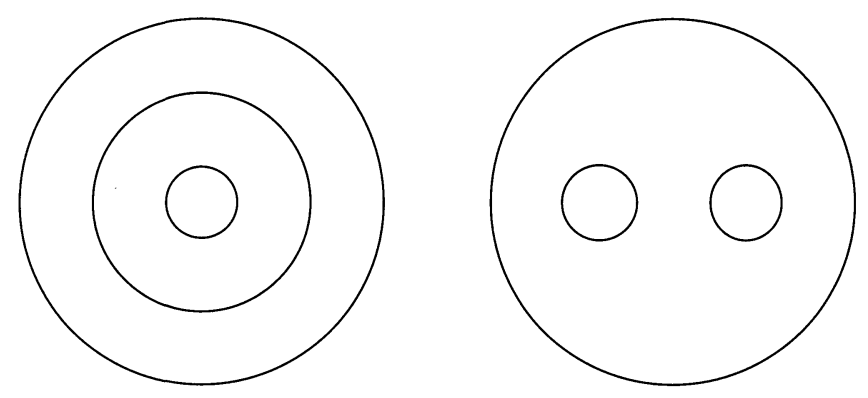

FIGURE 2. A dartbord and a pig nose.

We know that the images of the intervals $(-\infty, 0)$ and $(0,1)$ are circles. On the other hand, in a sufficiently small neighborhood of $x=0$ in the upper half-plane, the map $s_{0}$ can be approximated by the power function $x^{\lambda}$ as closely as we like. So we conclude that any sufficiently small neighborhood of $x=0$ in the upper half-plane is mapped under $s_{0}$ onto the ring of radii $\exp \left(-\theta_{0} \pi\right)$ and 1 ; the unit circle is the image of the interval $(0,1)$.

In the same way, any sufficiently small neighborhood of $x=1$ of the upper half-plane is mapped under $s_{1}$ to the ring of radii 1 and $\exp \left(\theta_{1} \pi\right)$; the unit circle is the image of the interval $(0,1)$. This is because the variable $1-x$ is real positive on the interval $(0,1)$.

We would like to draw these two rings in the same plane. Note that, since $a+b-c$ is purely imaginary, 
we have $\bar{a}=1-a, \bar{b}=1-b$, and $\bar{c}=2-c$, and that, since the Gamma function is a real function, $A=\bar{D}$ and $B=\bar{C}$ Now it is easy to show the following.

Lemma 3.1. If $s_{0}$ moves along the circle of radius $r$, then $s_{1}$ moves along the circle with center $K$ and radius $R$, where

$$
K=-\frac{\xi\left(1-r^{2}\right)}{|\xi|^{2}-r^{2}}, \quad R=\frac{\left.r|1-| \xi\right|^{2} \mid}{\left.|| \xi\right|^{2}-r^{2} \mid} \quad \text { with } \quad \xi=\frac{\bar{A}}{B} .
$$

Thus the unit circle is mapped to the unit circle, and the circle in the $s_{0}$-plane of radius

$$
r=\exp \left(-\theta_{0} \pi\right)
$$

is mapped to the circle in the $s_{1}$-plane with the center and the radius given just above. The following proposition describes the arrangement of the three circles. To make figure-drawing easier, we assume that the three purely imaginary exponents have positive imaginary parts.

\section{Proposition 3.2.}

$$
|K|-R-1>0, \quad \exp \left(\theta_{1} \pi\right)-|K|-R>0 .
$$

Proof. Recall the well-known formula

$$
\Gamma(z) \Gamma(1-z)=\frac{\pi}{\sin \pi z},
$$

which implies in particular

$$
\left|\Gamma\left(\frac{1}{2}+i y\right)\right|^{2}=\frac{\pi}{\cosh \pi y}, \quad \text { for } y \text { real. }
$$

On the other hand, by the definition of $\xi$, we have

$$
|\xi|=\left|\frac{\Gamma(a)}{\Gamma\left(a+i \theta_{0}\right)} \frac{\Gamma(b)}{\Gamma\left(b+i \theta_{0}\right)}\right| .
$$

Since the real parts of $a$ and $b$ are $\frac{1}{2}$, we can apply the formula above to get

$$
\begin{aligned}
|\xi|^{2} & =\frac{\cosh \left(\left(-\theta_{0}+\theta_{1}+\theta_{2}\right) \pi / 2\right) \cosh \left(\left(\theta_{0}-\theta_{1}+\theta_{2}\right) \pi / 2\right)}{\cosh \left(\left(\theta_{0}+\theta_{1}+\theta_{2}\right) \pi / 2\right) \cosh \left(\left(\theta_{0}+\theta_{1}-\theta_{2}\right) \pi / 2\right)} \\
& =\frac{\cosh \theta_{2} \pi+\cosh \left(\theta_{0}-\theta_{1}\right) \pi}{\cosh \theta_{2} \pi+\cosh \left(\theta_{0}+\theta_{1}\right) \pi} .
\end{aligned}
$$

So we can conclude that $|\xi|$, as a function of $\theta_{2} \geq 0$, increases monotonically to 1 and that

$$
1>|\xi|_{\theta_{2}=0}=\frac{\exp \left(\theta_{1}-\theta_{0}\right) \pi+1}{\exp \left(-\theta_{0} \pi\right)+\exp \left(\theta_{1} \pi\right)}>r\left(=e^{-\theta_{0} \pi}\right) .
$$

Now we are ready to prove the proposition using the identities

$$
\begin{aligned}
|K|-R-1 & =\frac{\left(1-r^{2}\right)|\xi|-\left.r|1-| \xi\right|^{2}|-||\xi|^{2}-r^{2} \mid}{\left.|| \xi\right|^{2}-r^{2} \mid} \\
& =\frac{\left(1-r^{2}\right)|\xi|-r\left(1-|\xi|^{2}\right)-\left(|\xi|^{2}-r^{2}\right)}{\left(|\xi|^{2}-r^{2}\right)} \\
& =\frac{(1-r)(|\xi|-r)(1-|\xi|)}{\left(|\xi|^{2}-r^{2}\right)}, \\
e^{\theta_{1} \pi}-|K|-R & =e^{\theta_{1} \pi}-\frac{|\xi|\left(1-r^{2}\right)}{|\xi|^{2}-r^{2}}-\frac{r\left(1-|\xi|^{2}\right)}{|\xi|^{2}-r^{2}} \\
& =e^{\theta_{1} \pi}-\frac{(1-r|\xi|)(|\xi|+r)}{|\xi|^{2}-r^{2}} \\
& =\frac{e^{\theta_{1} \pi}(|\xi|-r)-1+r|\xi|}{|\xi|-r} \\
& =\frac{\left(e^{\theta_{1} \pi}+e^{-\theta_{0} \pi}\right)|\xi|-\left(e^{\left(\theta_{1}-\theta_{0}\right) \pi}+1\right)}{|\xi|-r} .
\end{aligned}
$$

Proposition 3.3. Let the three exponents be strictly imaginary. Then the image of the upper half $x$ plane under the Schwarz map $s_{1}$ is bounded by the three disjoint circles: the unit circle with center 0 , the circle of radius $R$ with center $K$, and the circle of radius $\exp \left(\theta_{1} \pi\right)$ with center 0 . The third one encircles the other two, making a pig nose.

Now rotate the $s_{1}$-plane so that the second circle has its center at $-|K|$. More precisely, define a new Schwarz map $s$ as

$$
s: x \longmapsto \frac{\bar{\xi}}{|\xi|} s_{1}(x) .
$$

Then the three circles are as in Figure 3. Denote

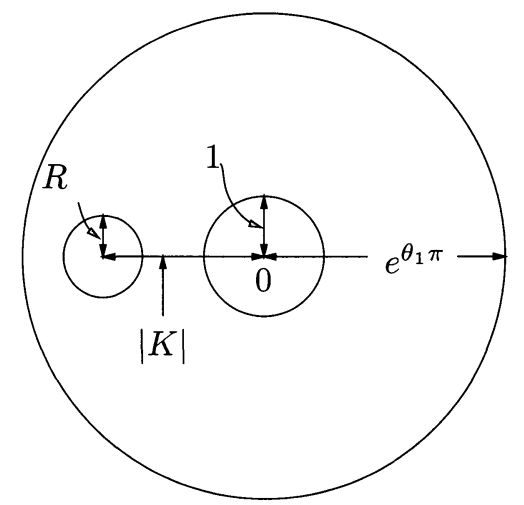

FIGURE 3. Three circles showing the pig nose PN. 

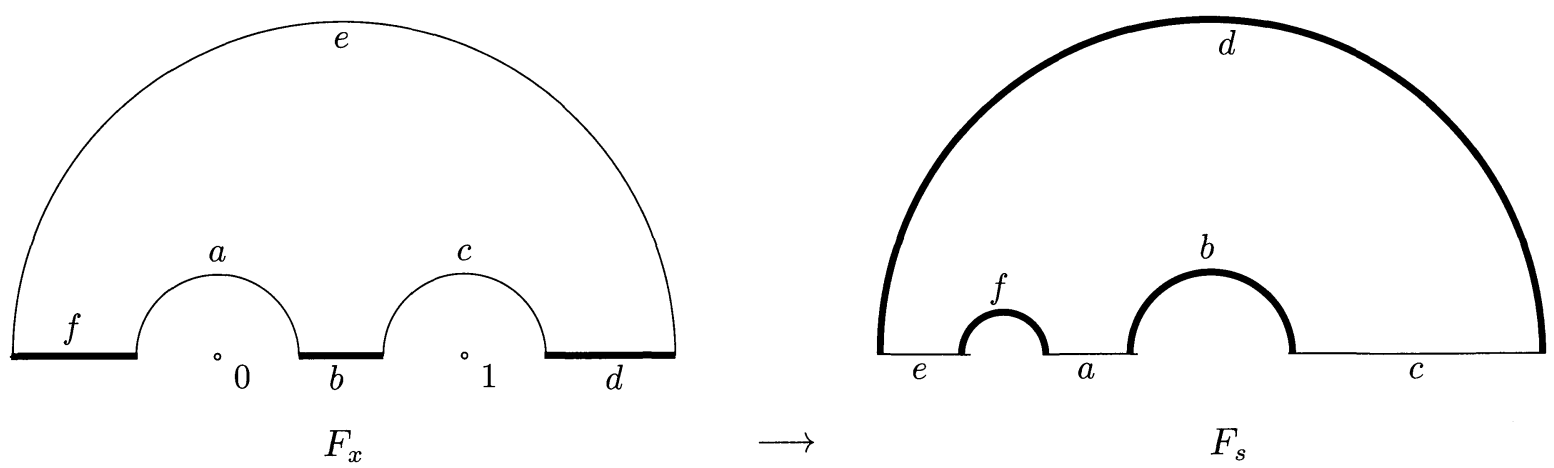

FIGURE 4. The fundamental domains $F_{x}$ and $F_{s}$

by $\mathcal{P N}$ the complement of the two small discs in the big disc, and call it the pig nose.

Remark 3.4. The proposition does not mean that the image of the upper half-plane under $s$ is $\mathcal{P N}$. The image covers the whole plane infinitely many times. Refer to Section 4B.

\section{FUNDAMENTAL DOMAINS}

Our Schwarz map $s$ is doubly multivalued, that is, $\infty$-to- $\infty$. The restricted map, as in the last subsection, on the upper half $x$-plane is still $\infty$-to-one. We find domains $F_{x}$ and $F_{s}$ so that the restriction of the Schwarz map on $F_{x}$ gives a one-to-one correspondence between $F_{x}$ and $F_{s}$, and that the restricted map reproduces the whole Schwarz map due to the reflection principle.

Now cut the pig nose $\mathcal{P \mathcal { N }}$ along the real axis; the upper half part with two-arched bridge shape will be denoted by $F_{s}$. Its inverse image will be denoted by $F_{x}$. Though this domain also has the shape of a twoarched bridge, it is a little distorted, being bounded by three real intervals and three curves (Figure 4).

Anyway, these two domains will be called fundamental domains for $s$.

\section{A. Analytic Continuation I}

We now see what happens if we repeatedly apply the Schwarz reflection principle to the restricted map

$$
\left.s\right|_{F_{x}}: F_{x} \longrightarrow F_{s}
$$

through the three real intervals (the intersection of the real axis and the closure of $F_{x}$ ) to the (complex conjugate) mirror image $\bar{F}_{x}$ of $F_{x}$. Since the images of the three intervals are semicircles, each image of $\bar{F}_{x}$ can be known by the reflection with respect to one of these circles, as in Figure 5. Next we apply the reflection principle again through the three intervals, and so on. Eventually we get:

Proposition 4.1. The whole image will cover the upper half s-plane $\mathbb{H}_{s}$. The inverse map, defined on $\mathbb{H}_{s}$, is single-valued, covers infinitely many times the 2-connected domain $F_{x} \cup \bar{F}_{x} \cup\{$ the three intervals\}, and gives the isomorphism

$$
\mathbb{H}_{s} / \Lambda \cong F_{x} \cup \bar{F}_{x} \cup\{\text { the three intervals }\},
$$

where $\Lambda$ is the monodromy group of the hypergeometric equation, forming a so-called Schottky group.

Remark 4.2. The shape of $F_{s}$ tells us that $\Lambda$ and $s^{-1}$ tend (see [Ichikawa and Yoshida 2001]) to $\Gamma(2)$ and $\lambda$, respectively, where $\Gamma(2)$ is the elliptic principal congruence subgroup of level 2 and $\lambda$ is the Lambda function.

Our Schottky group $\Lambda=\Lambda(a, b, c)$ defines a curve (Riemann surface) of genus 2 ,

$R=R(a, b, c):=$ (domain of discontinuity) $/ \Lambda(a, b, c)$

Since every such curve is hyperelliptic, our $R$ must be the double cover of the projective line $\mathbb{P}^{1}$ branching at six points. It is natural to ask then whether we can find the six points; these six points will tell

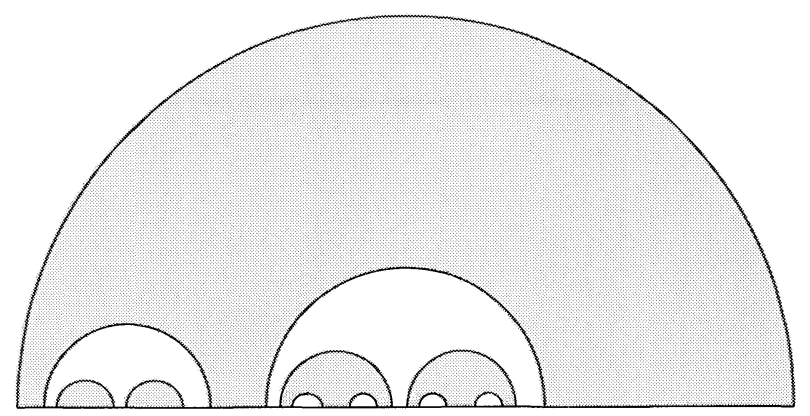

FIGURE 5. Action of a Schottky group on $\mathbb{H}_{s}$. 


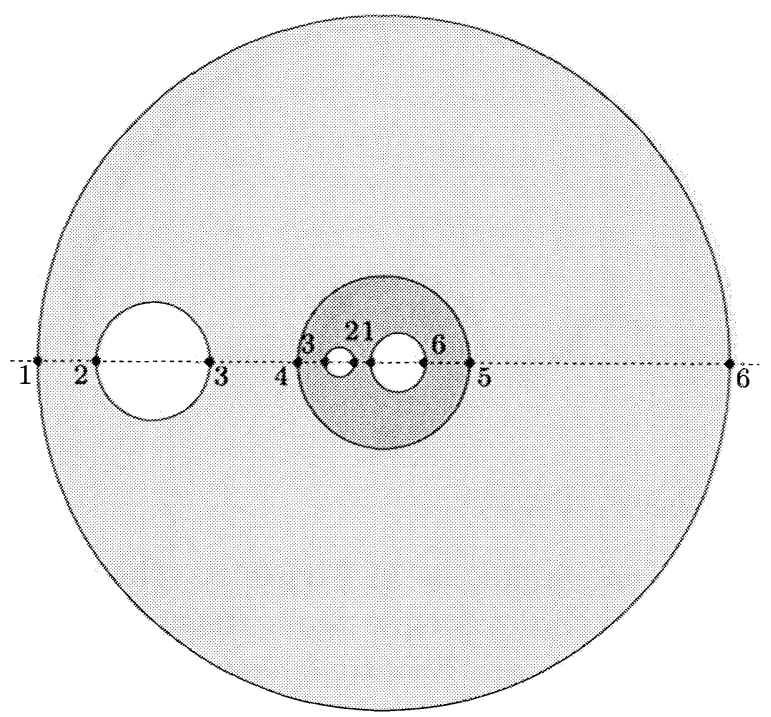

FIGURE 6. The curve of genus 2 .

where our real 3-dimensional family of curves lives in the whole (complex 3-dimensional) moduli space of curves of genus 2 . The double cover $R \rightarrow \mathbb{P}^{1}$ defines a holomorphic involution with exactly six fixed points. An explicit expression of this projection will be given elsewhere.

Reflect the pig nose $\mathcal{P N}$ with respect to the unit circle, and we get a small pig nose $p n$ in the unit disc. The Riemann surface $R$ can be obtained by identifying the two pairs of corresponding circles bounding the domain

$$
R^{\circ}=\mathcal{P \mathcal { N }} \cup p n .
$$

Let inv be the composition of the two reflections defined on $R^{\circ}$ with respect to the real axis and the unit circle. Since the axis and the circle intersect perpen- dicularly, these two reflections are commutative and so the composite defines a holomorphic involution on $R^{\circ}$ and so it induces an involution on $R$. The fixed points of inv consists of six points in $R^{\circ}$ : the intersections of the real axis and the three circles bounding pn (Figure 6). We already know the exact coordinates of these six points by Lemma 3.1.

\section{B. Analytic Continuation II}

We continue analytically the restricted map $\left.s\right|_{F_{x}}$ : $F_{x} \mapsto F_{s}$ to the upper half $x$-plane $\mathbb{H}_{x}$, and obtain one of the main results of this paper:

Proposition 4.3. If we apply the reflection principle to the inverse map of $\left.s\right|_{F_{x}}$ through the real intervals (the intersection of the real axis and the closure of $\left.F_{s}\right)$ to the (complex conjugate) mirror image $\bar{F}_{s}$ of $F_{s}$, back to $F_{s}$ through the three intervals, and continue as we did in the $x$-space in the previous section, then the whole image under the inverse map, namely $s^{-1}(\mathcal{P N})$, does not cover the entire half-plane $\mathbb{H}_{x}$ but is the complement of infinitely many disjoint (topological) discs in $\mathbb{H}_{x}$.

Sketch of proof. We apply the reflection principle for $s$ along the curve $c$ bounding $F_{x}$ and the line segment $c$ bounding $F_{s}$. See Figure 4 and its detail in Figure 7.

The line segments $b$ and $d$ are prolonged toward 1 and the semicircles $b$ and $d$ are prolonged to the lower $s$-space. The inverse image under $s$ of the line segment $e$ has one end on the line segment $d$ and the other end in $H_{x}$, not on the real axis. This is because the intervals $(0,1)$ and $(1,+\infty)$ are mapped to the circles $b$ and $d$, respectively, and so no part
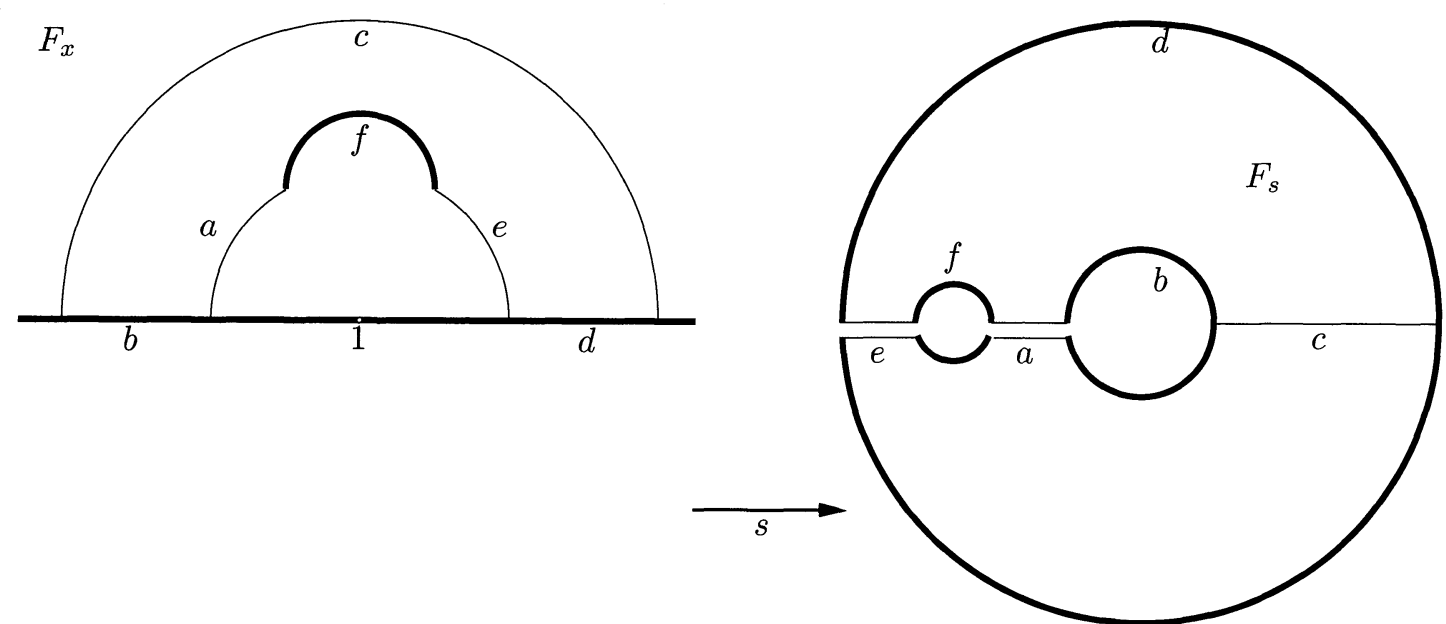

FIGURE 7. Analytic continuation through the curve/segment $c$. 


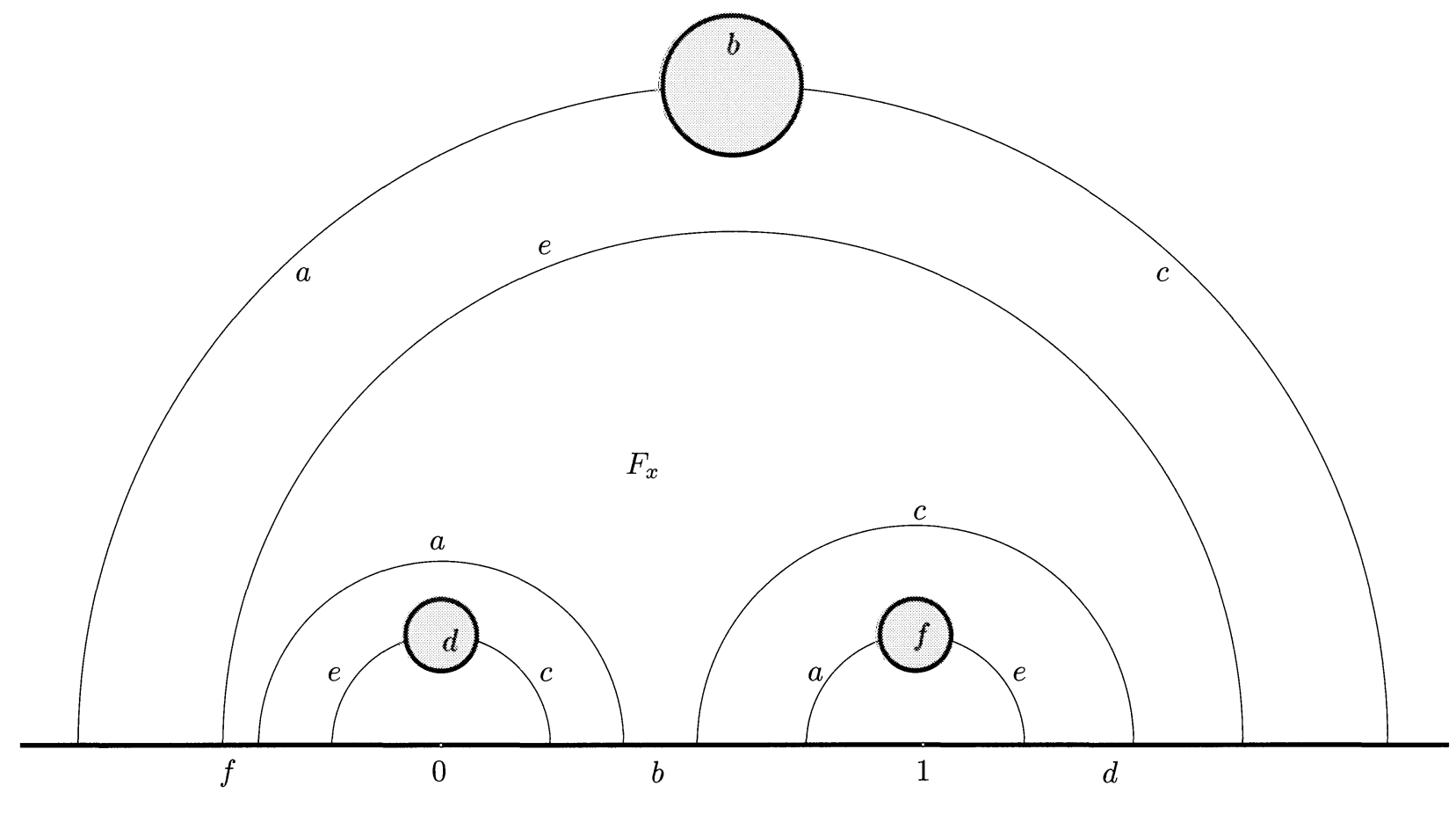

FIGURE 8. The inverse image of $\mathcal{P} \mathcal{N}$.

of these interval can be mapped to the other circle $f$. Similarly the inverse image of the line segment $a$ has one end on the line segment $b$ and the other end in $H_{x}$. (This is the essential difference from the Schottky case appeared in the previous subsection.) The completion of the proof is now immediate.

The proposition above can be paraphrased as follows: the upper half-plane $\mathbb{H}_{x}$ covers under the map $s$ the whole $s$-sphere infinitely many times, and the three real intervals bounding $\mathbb{H}_{x}$ are mapped to the three circles. (See Figure 8.)

\section{NUMERICAL EXPERIMENTS}

As we know, the fundamental domain $F_{s}$ is bounded by three line segments and three semicircles, and the fundamental domain $F_{x}$ is bounded by three line segments and three curves. None of these curves can be a part of any circle. Indeed, if one of a curve is a part of a circle, it must be a semicircle, and by symmetry, all the three curves must be semicircles; then the inverse map $F_{s} \rightarrow F_{x}$ has exactly the same properties as $s$; This implies the inverse of a Schwarz map must be also a Schwarz map; of course, this can not happen as we saw in the previous subsection.
We are interested in the shape of the three curves bounding $F_{x}$. We show how they are near to and distorted from circles by numerical experiments.

Since these three curves can be equally treated, we mainly consider the boundary curve $\mathcal{F S C}$ (read fake semicircle) surrounding the point $x=1$. The $s$-map is given by

$$
s(x)=\frac{\eta}{|\eta|}(1-x)^{c-a-b} \frac{F_{1}}{F_{0}},
$$

where

$$
\begin{aligned}
\eta & =\frac{\Gamma(a+b-c) \Gamma(1-a) \Gamma(1-b)}{\Gamma(a-c+1) \Gamma(b-c+1) \Gamma(c-a-b)}, \\
F_{0} & =F(a, b, a+b-c+1 ; 1-x), \\
F_{1} & =F(c-a, c-b, c-a-b+1 ; 1-x) .
\end{aligned}
$$

We use the variable

$$
w=1-x
$$

and fix the branch of the power function

$$
(1-x)^{c-a-b} \text { by } \exp ((c-a-b)(\log |w|-\arg (w) i)) \text {. }
$$

\section{A. Fake Semicircles for Several $\left[\theta_{0}, \theta_{1}, \theta_{2}\right]$}

For several choices of parameters, we draw the shape of the fake semicircles $\mathcal{F S C}$ surrounding the origin $w=0$. Surprisingly enough, they all look like circles 

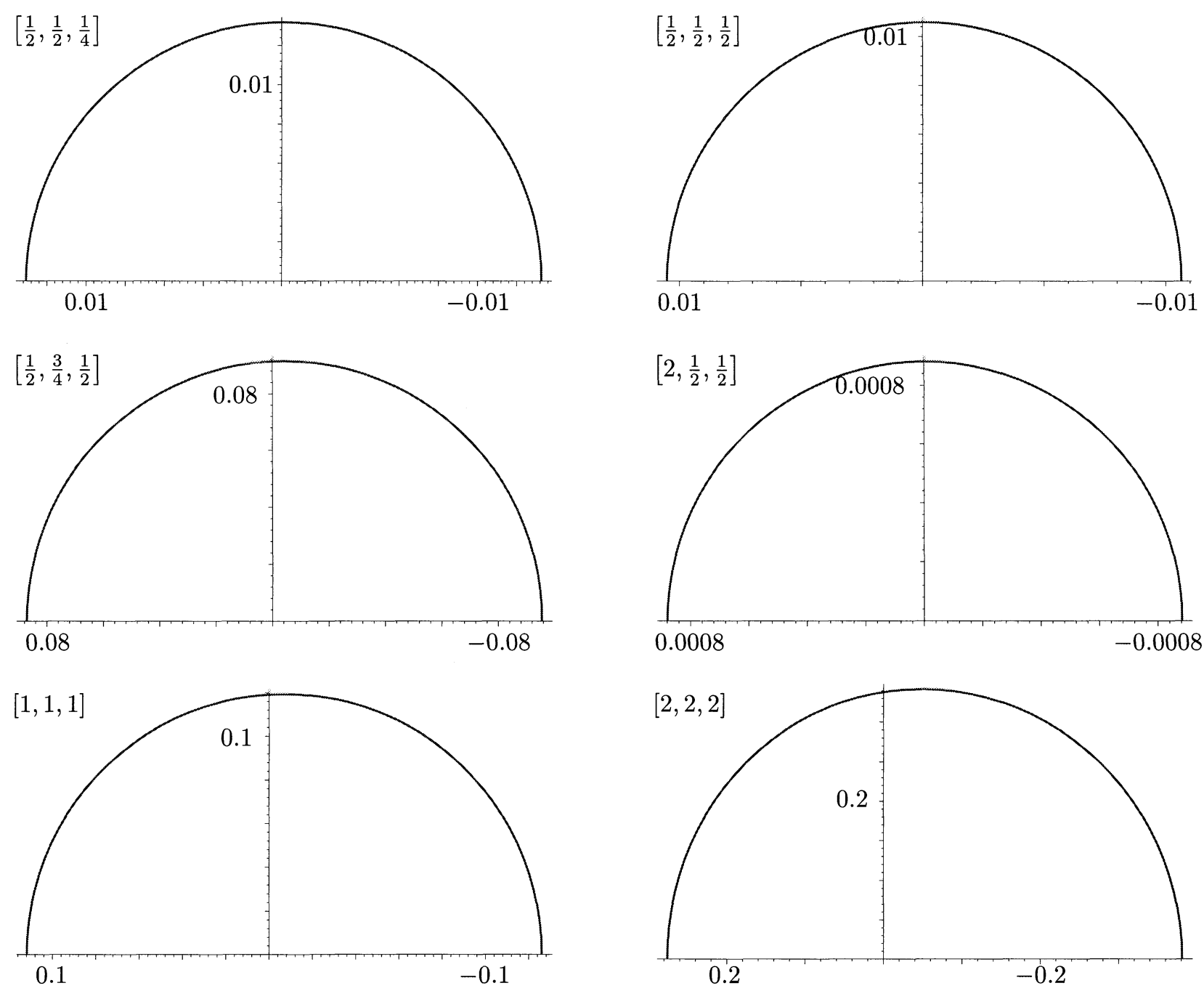

FIGURE 9. Fake semicircles in $w$ complex plane ( $\operatorname{Re} w$ horizontal). The triple labeling each panel is $\left[\theta_{0}, \theta_{1}, \theta_{2}\right]$.

(see Figure 9), though the size and the position of "center" varies much depending on the parameters.

5B. Fake semicircle for $\left[\frac{1}{2}, \frac{1}{2}, \frac{1}{2}\right]$

We obtain here a precise shape for the symmetric case $\left[\theta_{0}, \theta_{1}, \theta_{2}\right]=\left[\frac{1}{2}, \frac{1}{2}, \frac{1}{2}\right]$. The shape of the domain $F_{s}$ can be seen by

$$
e^{\theta_{1} \pi} \approx 4.8, \quad|K| \approx 2.3, \quad R \approx 0.76
$$

The rough shape of $F_{x}$ is shown in Figure 10.

The whole fake circle $\mathcal{F C}$, the connected component of

$$
\{w=1-x: \operatorname{Im} s(x)=0\}
$$

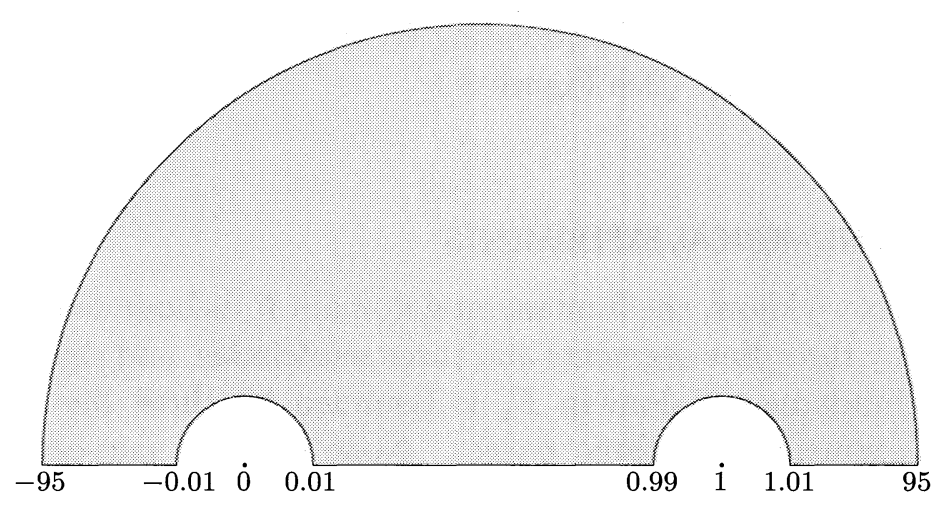

surrounding the origin $w=0$, looks like a circle (see Figure 11).

Since the images under $s$ of the intervals $-\infty<$ $w<0$ and $0<w<1$ are genuine circles (thanks to

Schwarz's reflection principle), the fake circle $\mathcal{F C}$ is symmetric with respect to the real axis; the upper half is the fake semicircle $\mathcal{F S C}$. 


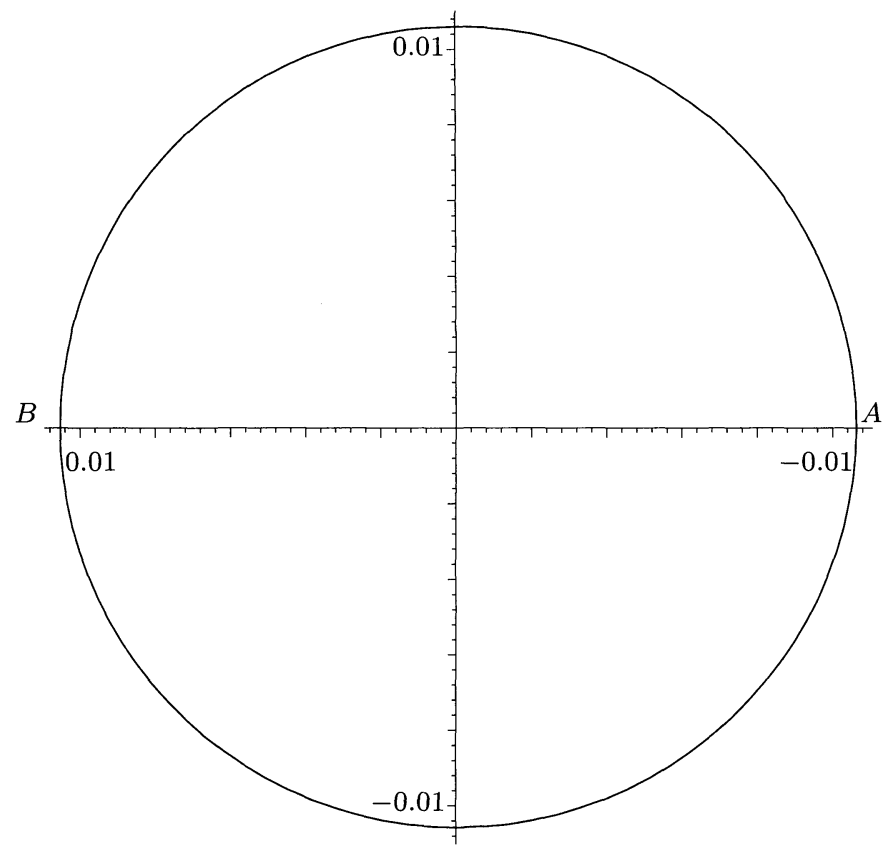

FIGURE 11. The fake circle $\mathcal{F}$.

Due to this shape, we can restrict the variable $w$ to the area $\left\{|w|<\frac{2}{100}\right\}$. Define polynomials $\varphi_{0}$ and $\varphi_{1}$ of degree 15 that are truncations of $F_{0}$ and $F_{1}$, respectively. Since

$$
\begin{aligned}
& \left|\frac{(a+k)(b+k)}{(a+b-c+1+k)(1+k)}\right|<1, \\
& \left|\frac{(c-a+k)(c-b+k)}{(c-a-b+1+k)(1+k)}\right|<1
\end{aligned}
$$

for any $k \geq 0$, we have

$$
\left|\varphi_{0}(w)-F_{0}(w)\right|<10^{-25}, \quad\left|\varphi_{1}(w)-F_{1}(w)\right|<10^{-25}
$$

for $|w|<\frac{2}{100}$; similarly,

$$
\frac{96}{100}<\left|F_{0}\right|, \quad\left|F_{1}\right|<\frac{100}{98} .
$$

Since

$$
\exp (-\pi / 2) \leq\left|\frac{\eta}{|\eta|} w^{i / 2}\right| \leq \exp (\pi / 2)
$$

we have

$$
10^{-2}<|s(x)|<10^{2}
$$

Now define

$$
t(w)=\frac{\eta}{|\eta|} w^{i / 2} \frac{\varphi_{0}}{\varphi_{1}}
$$

and let $t_{c}(w)$ be numerical value of $t(w)$ to the precision $10^{-20}$. Summing up the estimates above, we see that

$$
\left|s-t_{c}\right| \leq 10^{-17}
$$

for $w$ with $|w|<\frac{2}{100}$.

Let $A=\left(l_{u}, 0\right)$ and $B=\left(r_{u}, 0\right)$ denote the rightmost and the leftmost points of the fake circle $\mathcal{F C}$ on the $u$-axis as shown in Figure 11. By a numerical experiment, we get the estimate

$$
\begin{aligned}
-0.0106390151470973 & \leq l_{u} \leq-0.0106390151470972 \\
0.0105270180426873 & \leq r_{u} \leq 0.0105270180426874 .
\end{aligned}
$$

Let $C=\left(m_{u}, 0\right)$ be the midpoint of the line segment $A B$; then, $m_{u}=-0.000055998552205 \pm 10^{-14}$. The length $\overline{A C}=\overline{B C}$ is

$$
\operatorname{rad}=0.01058301659489\left( \pm 10^{-14}\right) .
$$

Compute the $v$-coordinate ht of the point on the curve with real value $m_{u}$ :

$$
\text { ht }=0.01058323446332\left( \pm 10^{-14}\right) \text {. }
$$

Hence:

Fact. The fake circle $\mathcal{F C}$ is not a circle; it is a little longer in $v$-direction, though the difference is only

$$
\mathrm{rad} / \mathrm{ht}=0.9999794 \text {. }
$$

The distance $r$ between the midpoint $C$ and points on $\mathcal{F C}$ is plotted in Figure 12 as a function of the height $v$.

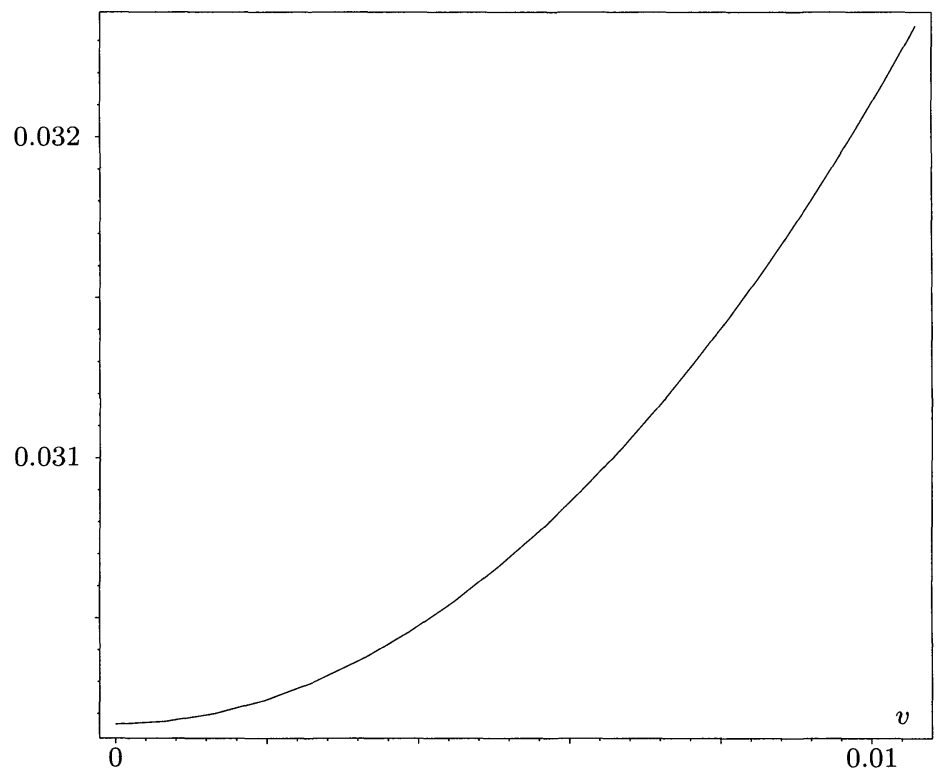

FIGURE 12. Distortion of the radii from $C$. The vertical axis represents $10^{4}(r-0.01058)$. 

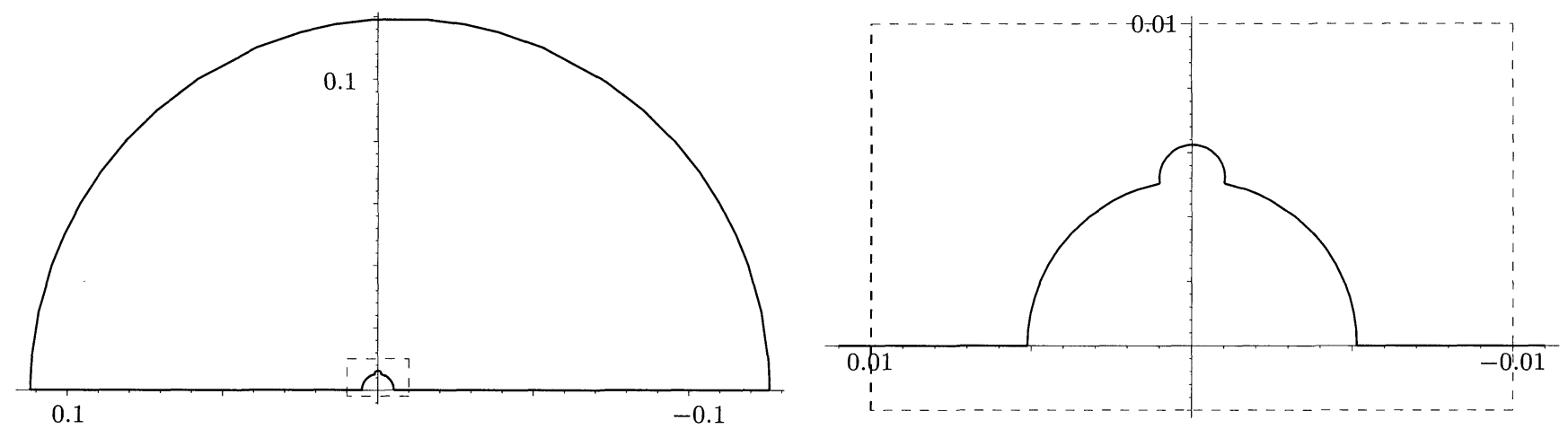

FIGURE 13. Left: The mirror image of $F_{x}$ through the the fake semicircle $c$. Right: Zoom near the origin.

Values of $\mathrm{rad} / \mathrm{ht}$ for other parameters are:

$$
\begin{array}{cc}
{\left[\theta_{0}, \theta_{1}, \theta_{2}\right]} & \mathrm{rad} / \mathrm{ht} \\
{\left[\frac{1}{2}, \frac{1}{2}, \frac{1}{4}\right]} & 0.9999705 \\
{\left[\frac{1}{2}, \frac{3}{4}, \frac{1}{2}\right]} & 0.9985123 \\
{\left[2, \frac{1}{2}, \frac{1}{2}\right]} & 1.0000005 \\
{[1,1,1]} & 0.9965072 \\
{[2,2,2]} & 0.9611695
\end{array}
$$

\section{C. A mirror image of the fundamental domain $F_{x}$}

Figure 13 is a numerically computed view of the left part of Figure 7, showing the mirror image of the fundamental domain $F_{x}$ with respect to the fake semicircle $c$. The parameters are $\left[\frac{1}{2}, \frac{1}{2}, \frac{1}{2}\right]$. Here we encounter again three very circle-like curves!

\section{CONCLUSION AND QUESTIONS}

Specialists expect that everything concerning the hypergeometric function should be computed explicitly (see, for example, Proposition 4.1 and the formulae in Section 2). We checked that $\mathcal{F} \mathcal{C}$ is not a circle. What is it? How can one describe the fake circle $\mathcal{F} \mathcal{C}$ ? Is this an algebraic curve, or maybe a trajectory of some differential equation? We hope that the numerical experiments we made here will help understand this misterious curve in the future.

\section{ACKNOWLEDGEMENTS}

We are grateful to Professor J. Wolfart, who kindly showed us the reference [Zapp 1994], which includes a summary of the papers of Schilling above. Yoshida thanks the Department of Mathematics, Kobe University, especially Professor K. Takano, for the warm hospitality.

\section{REFERENCES}

[Ichikawa and Yoshida 2001] T. Ichikawa and M. Yoshida, "Invariants of Schottky groups arising from the hypergeometric function", preprint 2001-9, Graduate School of Mathematics, Kyushu University, 2001.

[Iwasaki et al. 1991] K. Iwasaki, H. Kimura, S. Shimomura, and M. Yoshida, From Gauss to Painlevé: a modern theory of special functions, Aspects of mathematics E16, Vieweg, Wiesbaden, 1991.

[Schilling 1894] F. Schilling, "Beiträge zur geometrischen Theorie der Schwarzschen s-Funktion", Math. Annalen 44 (1894), 161-260.

[Schilling 1895] F. Schilling, "Die geometrischen Theorie der Schwarzschen $s$-Funktion für komplexe Exponenten", Math. Annalen 46 (1895), 62-76, 529-538.

[Yoshida 1997] M. Yoshida, Hypergeometric functions, my love, Aspects of mathematics E32, Vieweg, Wiesbaden, 1997.

[Zapp 1994] M. Zapp, Abbildungseigenschaften allgemeiner Dreiecksfunktionen, Diplomarbeit, Universität Frankfurt, 1994.

Takeshi Sasaki, Department of Mathematics, Kobe University, Kobe 657-8501 Japan (sasaki@math.kobe-u.ac.jp)

Masaaki Yoshida, Department of Mathematics, Kyushu University, Fukuoka 810-8560 Japan

(myoshida@math.kyushu-u.ac.jp) 